\title{
Erratum to: Molecular Mechanisms in Autoimmune Type 1 Diabetes: a Critical Review
}

\author{
Zhiguo Xie • Christopher Chang $\cdot$ Zhiguang Zhou
}

Published online: 30 May 2014

(C) Springer Science+Business Media New York 2014

Erratum to: Clinic Rev Allerg Immunol

DOI 10.1007/s12016-014-8422-2

The original version of this article unfortunately contained a mistake. The corresponding author should be:

\section{Zhiguang Zhou}

Diabetes Center, 2nd Xiangya Hospital, and Institute of Metabolism and Endocrinology, Key Laboratory of Diabetes Immunology, Ministry of Education, Central South University, National Clinical Research Center for Metabolic Diseases, Changsha, Hunan 410011, China

e-mail: zhouzg@hotmail.com

Thank you.

Christopher Chang, MD, PhD

Thomas Jefferson University

1600 Rockland Road

Wilmington, DE 19803

cchang@nemours.org

The online version of the original article can be found at http://dx.doi.org/ 10.1007/s12016-014-8422-2.

Z. Xie $\cdot$ Z. Zhou $(\bowtie)$

Diabetes Center, 2nd Xiangya Hospital, and Institute of Metabolism and Endocrinology, Key Laboratory of Diabetes Immunology,

Ministry of Education, Central South University, National Clinical

Research Center for Metabolic Diseases, Changsha, Hunan 410011,

China

e-mail: Zhouzg@hotmail.com

C. Chang

Division of Allergy and Immunology, Thomas Jefferson University,

Nemours/A.I. duPont Hospital for Children, 1600 Rockland Road,

Wilmington, DE 19803, USA

e-mail: cchang@nemours.org 